Supporting Information

\title{
Application of Two-Dimensional Nuclear Magnetic Resonance for Signal Enhancement by Spectral Integration Using a Large Dataset of Metabolic Mixtures
}

\author{
Takuma Misawa $^{1,2}$, Feifei Wei ${ }^{2}$, and Jun Kikuchi ${ }^{1,2,3^{*}}$ \\ ${ }^{1}$ Graduate School of Medical Life Science, Yokohama City University, 1-7-29 \\ Suehiro-cho, Tsurumi-ku, Yokohama, Kanagawa 230-0045, Japan. \\ ${ }^{2}$ RIKEN Center for Sustainable Resource Science, 1-7-22 Suehiro-cho, Tsurumi-ku, \\ Yokohama, Kanagawa 230-0045, Japan. \\ ${ }^{3}$ Graduate School of Bioagricultural Sciences, Nagoya University, 1 Furo-cho, \\ Chikusa-ku, Nagoya, Aichi 464-0810, Japan.
}

\section{Corresponding Author}

*Center for Sustainable Resource Science, 1-7-22 Suehiro-cho, Tsurumi-ku, Yokohama, Kanagawa 230-0045, Japan. Tel: +81455039439; Fax: +81455039489; Email:

jun.kikuchi@riken.jp 
Contents:

\section{$\underline{\text { Experimental parts }}$}

Figure S1. The scheme of the SENSI 2D function.

Figure S2. The line plots of coefficient of variation (CV) for A) KPi extracts and B) MeOD extracts. First, a SENSI 2D spectrum is generated by the integration of a number of randomly selected spectra as indicated in the X-axis from all the 1022 spectra data. Then, the $\mathrm{S} / \mathrm{N}$ ratio and the number of detected peaks of the SENSI 2D spectrum are calculated. This process is repeated for 10 times and the CVs of the $\mathrm{S} / \mathrm{N}$ ratios and the numbers of detected peaks of the 10 generated SENSI 2D spectra are calculated. Red: $\mathrm{CVs}$ of $\mathrm{S} / \mathrm{N}$ ratio; blue: $\mathrm{CVs}$ of number of detected peaks.

Figure S3. Assignment evidence. An expansion and signal annotation of A) Statistical total correlation spectroscopy (STOCSY) and B) ${ }^{1} \mathrm{H}_{-}{ }^{13} \mathrm{C}$ HSQC and ${ }^{1} \mathrm{H}_{-}{ }^{13} \mathrm{C}$ HSQC TOCSY spectrum of yellowfin goby (MeOD). The ${ }^{1} \mathrm{H}-{ }^{13} \mathrm{C}$ HSQC spectrum of yellowfin goby body muscle was performed in the phase-sensitive mode. The acquisition parameters were as follows: number of data points, 2048 for ${ }^{1} \mathrm{H}$ and 256 for ${ }^{13} \mathrm{C}$; spectral width, $9,804 \mathrm{~Hz}$ for ${ }^{1} \mathrm{H}$ and $26,410 \mathrm{~Hz}$ for ${ }^{13} \mathrm{C}$; mixing time, $60 \mathrm{~ms}$; acquisition time, $0.104 \mathrm{~s}$; delay time, $2.0 \mathrm{~s}$; dummy scan, 16; and number of scans, 48. The acquisition parameters of ${ }^{1} \mathrm{H}-{ }^{13} \mathrm{C}$ HSQC-TOCSY were as follows: number of data points, 1024 for ${ }^{1} \mathrm{H}$ and 256 for ${ }^{13} \mathrm{C}$; spectral width, 9,803 Hz for ${ }^{1} \mathrm{H}$ and $26,410 \mathrm{~Hz}$ for ${ }^{13} \mathrm{C}$; mixing time, $60 \mathrm{~ms}$; acquisition time, $0.052 \mathrm{~s}$; delay time, $1.5 \mathrm{~s}$; dummy scan, 16; and number of scans, 48. Ala, alanine; Gly, glycine; His, histidine; IMP, inosine monophosphate; Leu, leucine; Phe, phenylalanine; TMAO, trimethylamine N-oxide; Try, 
tryptophan; Val, valine.

Figure S4. The result of Kernel density estimation using the data of 1,022 individuals of yellowfin goby used in this study. The top $0.05 \%$ (big group, $\mathrm{n}=50$ ) and the bottom $0.05 \%$ (small group, $\mathrm{n}=50$ ) were selected as shown in the gray zones.

\section{The R script of SENSI 2D function}




\section{$\underline{\text { Experimental parts }}$}

Sample collection and preprocessing. Yellowfin gobies $(n=1022)$ were collected over a period of two years from May 2012 to November 2014 from the estuarine and coastal zones of 19 rivers in Japan. After measuring the body length, the fish samples were dissected. Body muscles were collected, lyophilized and crushed into powder.

NMR observation. For Kpi extraction, $5 \mathrm{mg}$ of each powdered sample was extracted with $700 \mu \mathrm{L}$ of $\mathrm{PBS}\left(0.1 \mathrm{M} \mathrm{K}_{2} \mathrm{HPO}_{4} / \mathrm{KH}_{2} \mathrm{PO}_{4}, \mathrm{pH} 7.0\right)$ containing $90 \%$ deuterium oxide with $1 \mathrm{mM}$ sodium 2,2-dimethyl-2-silapentane-5-sulfonate (DSS) internal standard at $65^{\circ} \mathrm{C}$ for 15 mins. For MeOD extraction, $30 \mathrm{mg}$ of each powdered sample was extracted with $1000 \mu \mathrm{L}$ of deuterated methanol (99.8\%, Cambridge Isotope Laboratories Inc., MA, USA) with $1 \mathrm{mM}$ sodium 2,2-dimethyl-2-silapentane-5-sulfonate (DSS) internal standard at $55^{\circ} \mathrm{C}$ for 15 mins. After centrifugation at $25^{\circ} \mathrm{C}$ for 5 mins, the extracted supernatant was transferred to a 5-mm NMR tube. Two dimensional $J$-resolved (2DJ) spectra (pulse sequence of jresgpprqf in Bruker BioSpin) were acquired at $298 \mathrm{~K}$ using a Bruker AVANCE II 700 spectrometer equipped with a ${ }^{1} \mathrm{H}$ inverse triple-resonance cryogenically cooled probe with Z-axis gradients (Bruker BioSpin GmbH, Rheinstetten, Germany). The acquisition time per spectrum was 4 mins. 


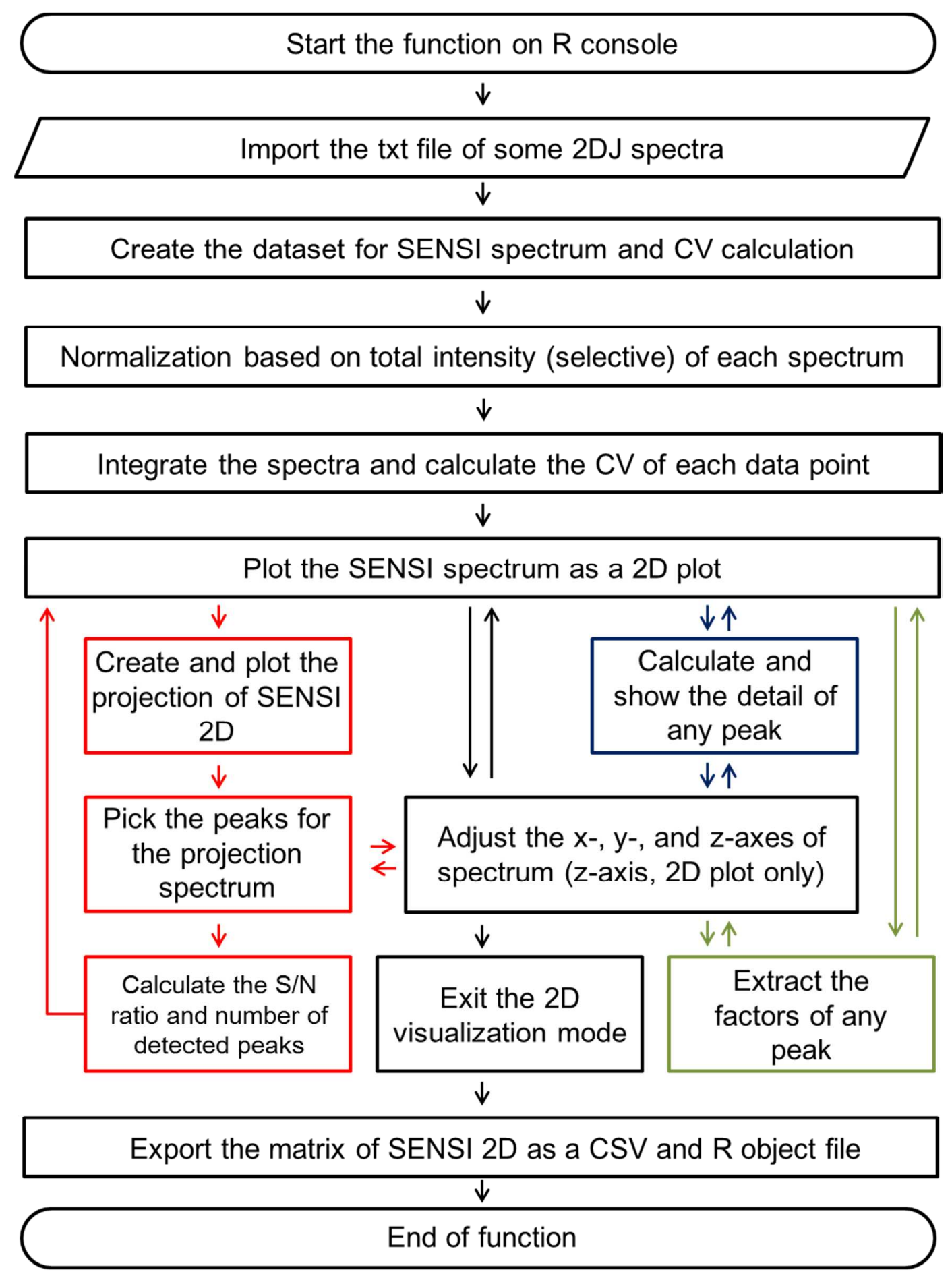

Figure S1. The scheme of the SENSI 2D function. 


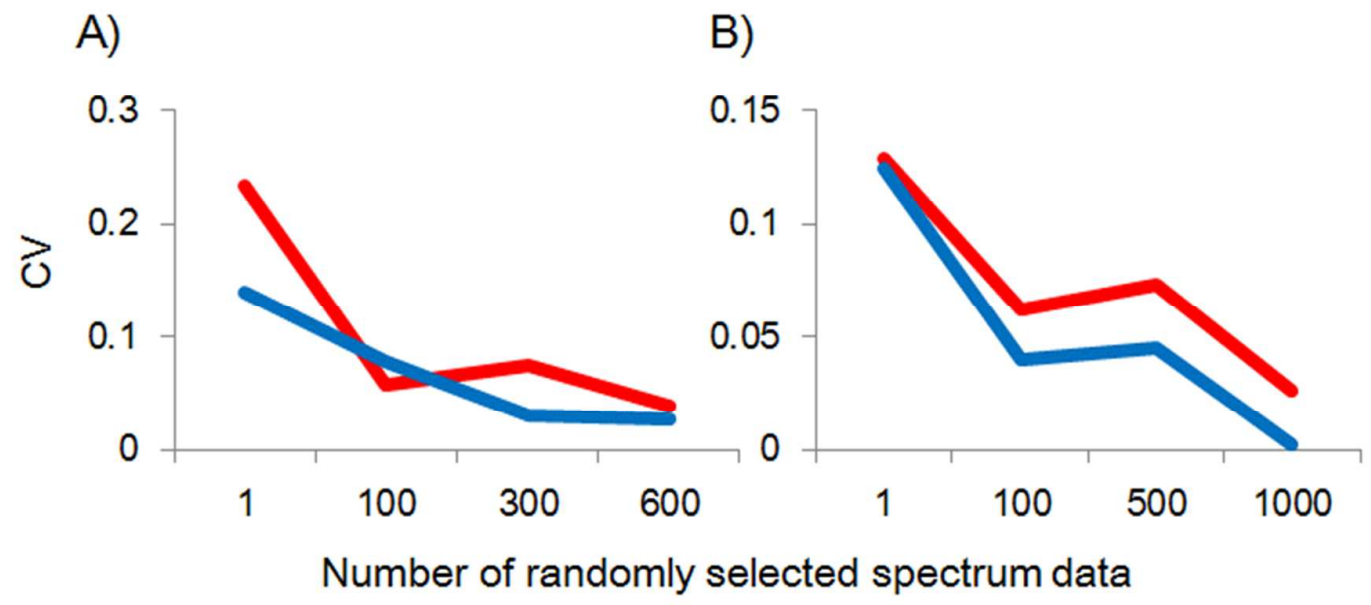

Figure S2. The line plots of coefficient of variation (CV) for A) Kpi extracts and B) MeOD extracts. First, a SENSI 2D spectrum is generated by the integration of a number of randomly selected spectra as indicated in the $X$-axis from all spectra data $(n=667$ for KPi extracts; $n=1022$ for MeOD extracts). Then, the $\mathrm{S} / \mathrm{N}$ ratio and the number of detected peaks of the SENSI 2D spectrum are calculated. This process is repeated for 10 times and the $\mathrm{CVs}$ of the $\mathrm{S} / \mathrm{N}$ ratios and the numbers of detected peaks of the 10 generated SENSI 2D spectra are calculated. Red: $\mathrm{CVs}$ of $\mathrm{S} / \mathrm{N}$ ratio; blue: $\mathrm{CVs}$ of number of detected peaks. 

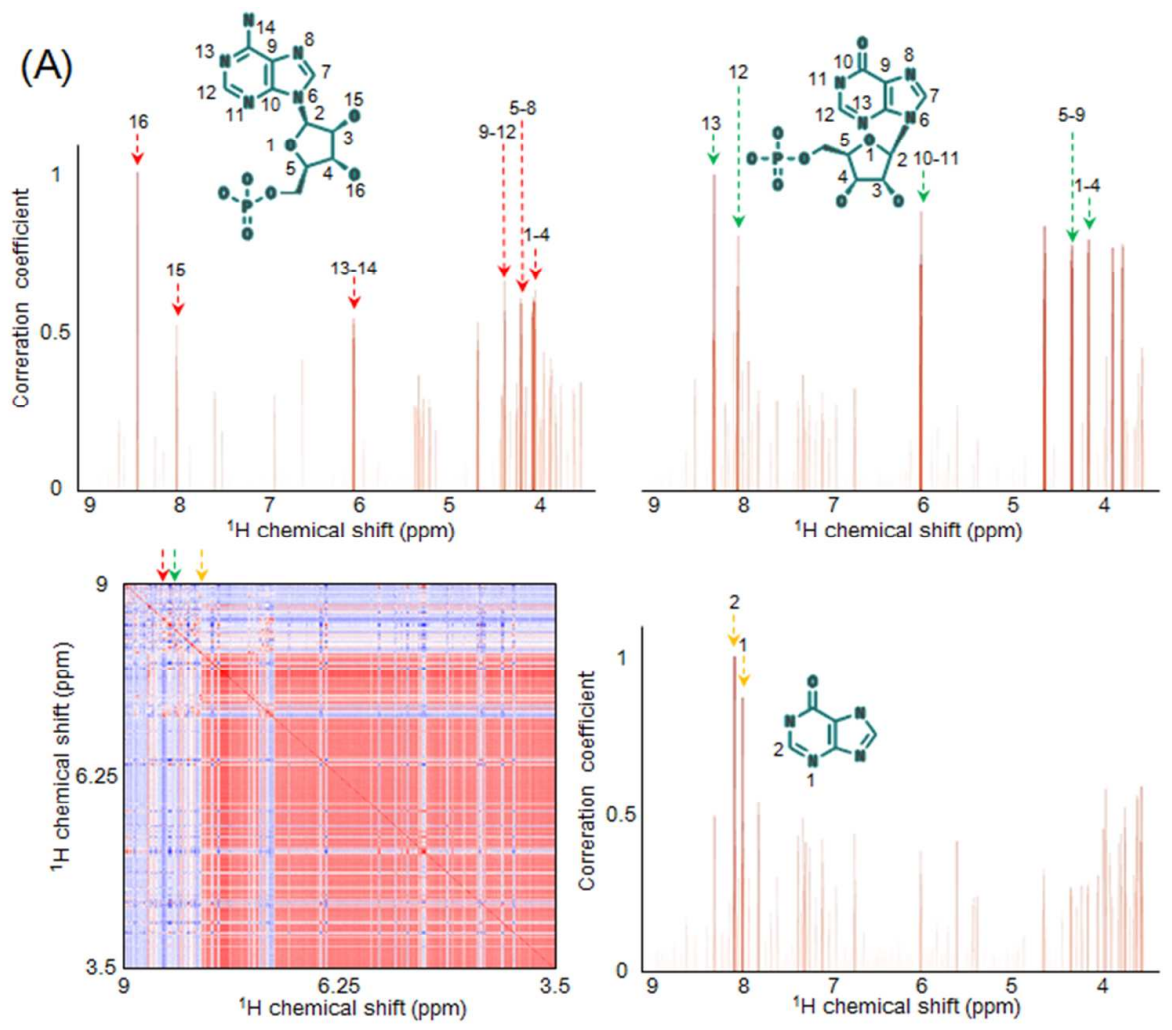

(B)

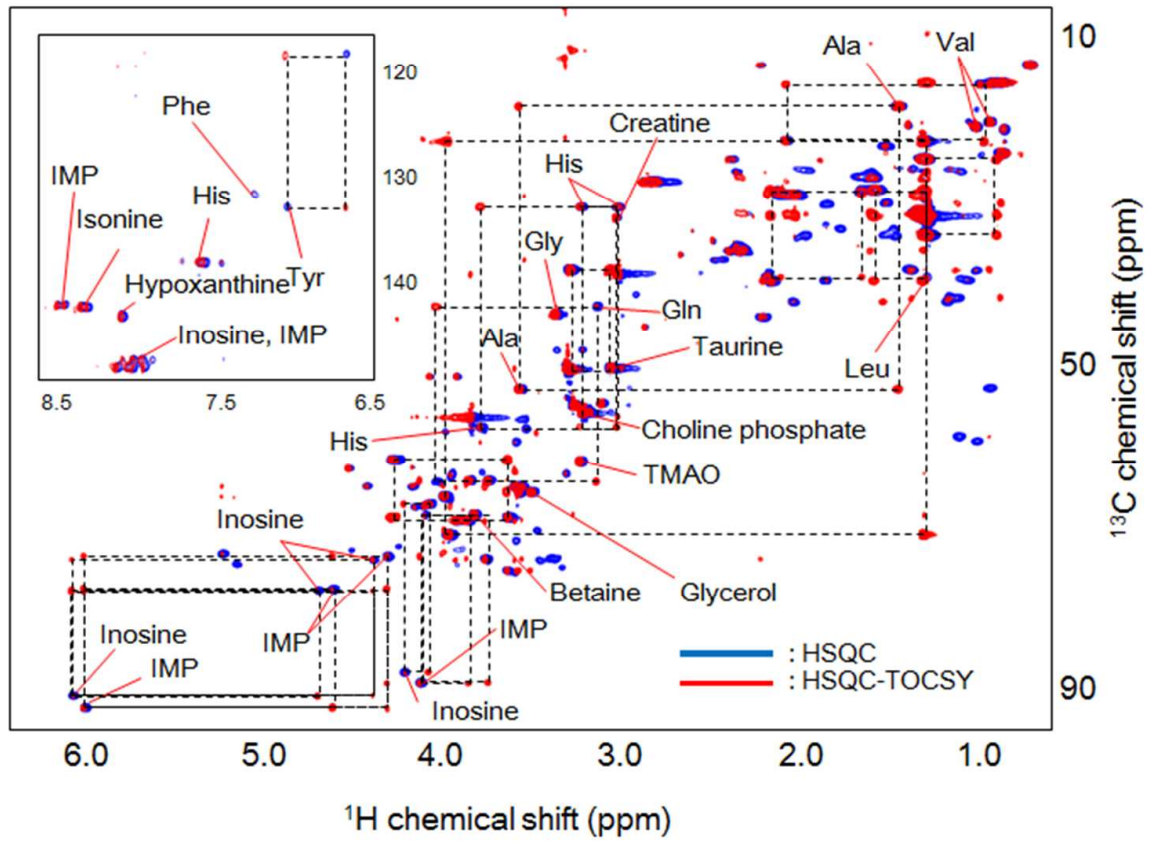

Figure S3. Assignment evidence. An expansion and signal annotation of A) Statistical 
total correlation spectroscopy (STOCSY) and B) ${ }^{1} \mathrm{H}^{-}{ }^{13} \mathrm{C}$ HSQC and ${ }^{1} \mathrm{H}_{-}{ }^{13} \mathrm{C}$ HSQC TOCSY spectrum of yellowfin goby (MeOD). The ${ }^{1} \mathrm{H}-{ }^{13} \mathrm{C}$ HSQC spectrum of yellowfin goby body muscle was performed in the phase-sensitive mode. The acquisition parameters were as follows: number of data points, 2048 for ${ }^{1} \mathrm{H}$ and 256 for ${ }^{13} \mathrm{C}$; spectral width, 9,804 $\mathrm{Hz}$ for ${ }^{1} \mathrm{H}$ and $26,410 \mathrm{~Hz}$ for ${ }^{13} \mathrm{C}$; mixing time, $60 \mathrm{~ms}$; acquisition time, $0.104 \mathrm{~s}$; delay time, $2.0 \mathrm{~s}$; dummy scan, 16; and number of scans, 48. The acquisition parameters of ${ }^{1} \mathrm{H}-{ }^{13} \mathrm{C}$ HSQC-TOCSY were as follows: number of data points, 1024 for ${ }^{1} \mathrm{H}$ and 256 for ${ }^{13} \mathrm{C}$; spectral width, 9,803 Hz for ${ }^{1} \mathrm{H}$ and $26,410 \mathrm{~Hz}$ for ${ }^{13} \mathrm{C}$; mixing time, $60 \mathrm{~ms}$; acquisition time, $0.052 \mathrm{~s}$; delay time, $1.5 \mathrm{~s}$; dummy scan, 16; and number of scans, 48. Ala, alanine; Gly, glycine; His, histidine; IMP, inosine monophosphate; Leu, leucine; Phe, phenylalanine; TMAO, trimethylamine N-oxide; Try, tryptophan; Val, valine. 


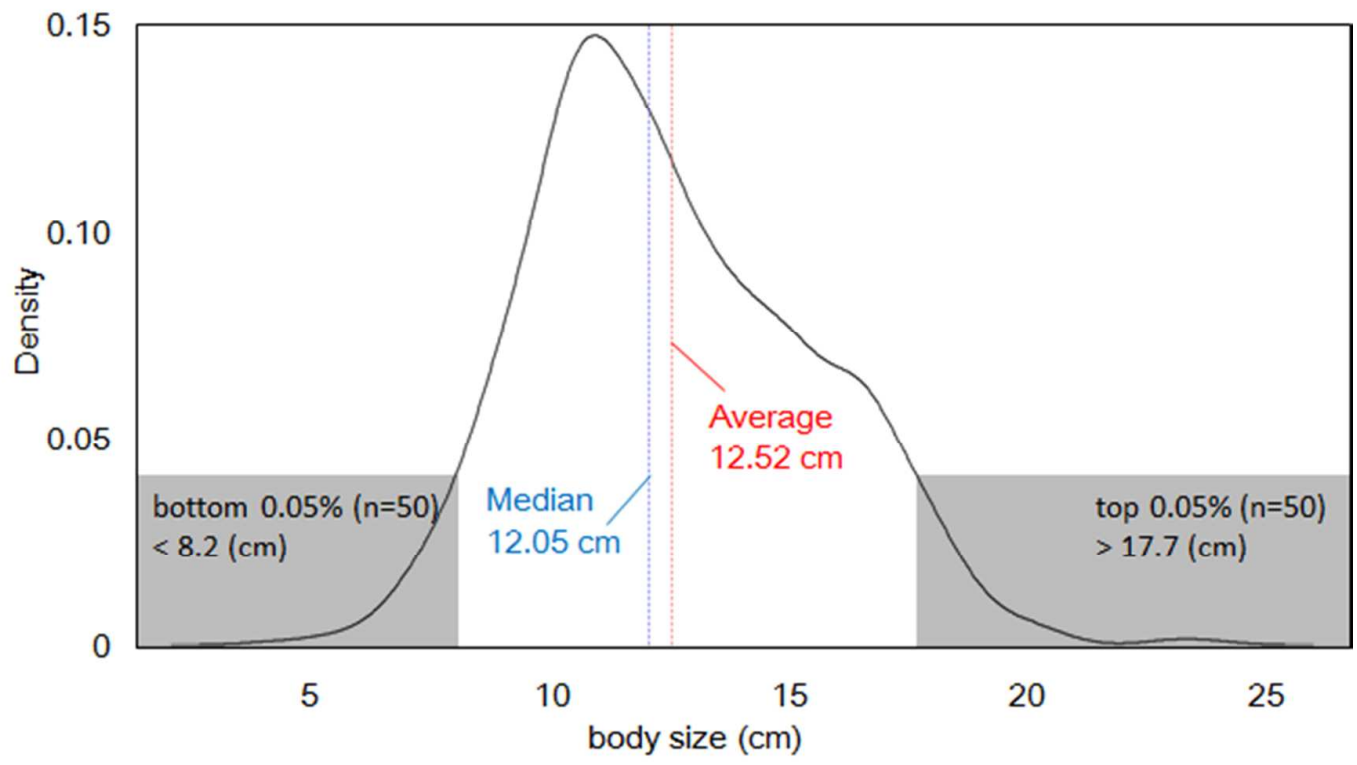

Figure S4. The result of Kernel density estimation using the data of 1,022 individuals of yellowfin goby used in this study. The top $0.05 \%$ (big group, $\mathrm{n}=50$ ) and the bottom $0.05 \%$ (small group, $\mathrm{n}=50$ ) were selected as shown in the gray zones. 


\section{The R script of SENSI 2D function}

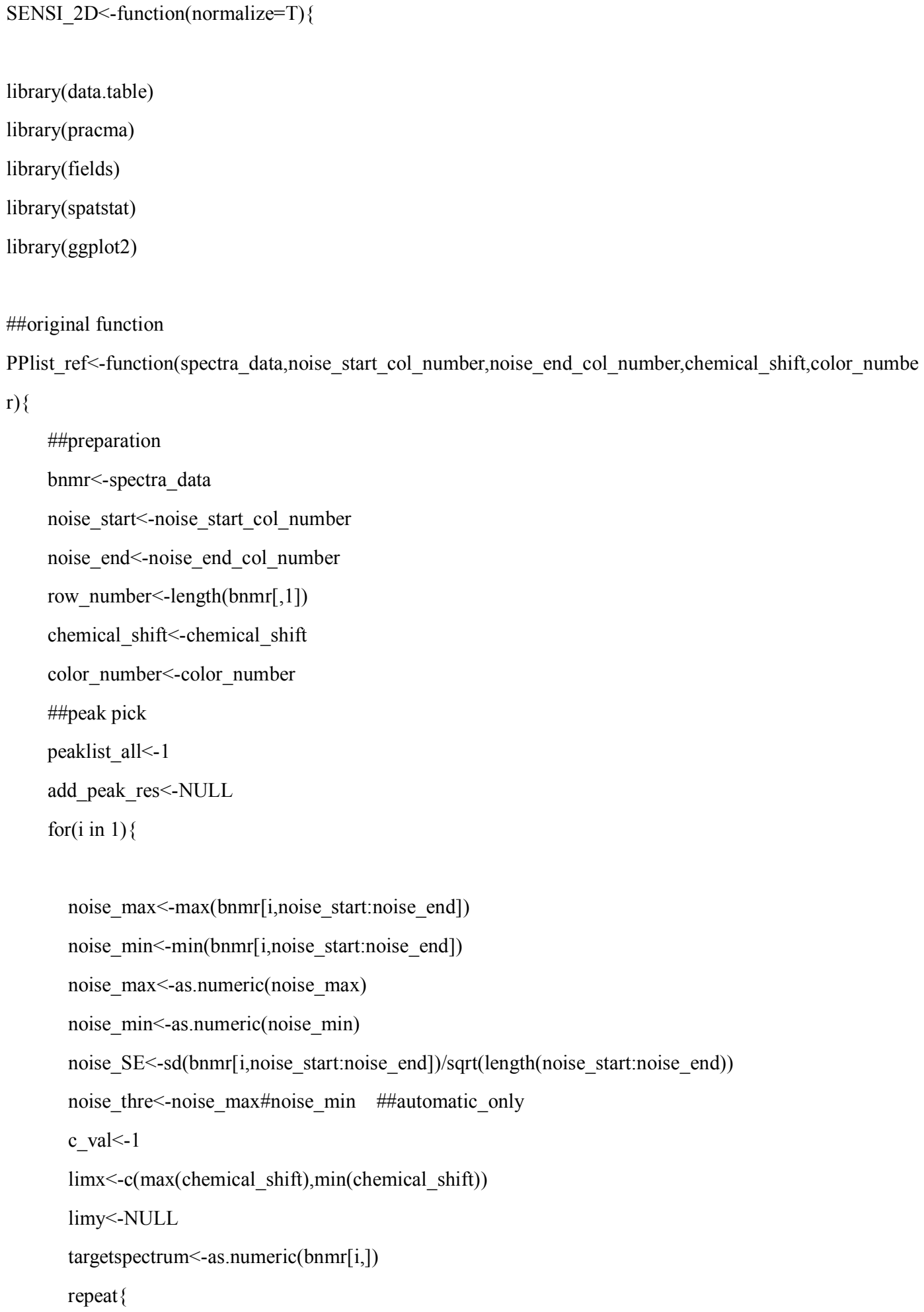


peaklist $<$-findpeaks(targetspectrum,nups $=1$, minpeakdistance $=1$, threshold $=$ noise_thre*c_val,npeaks $=$ length $(\operatorname{targ}$ etspectrum),sortstr=F,minpeakheight=noise_max)

title $<-$ "Auto pick mode on projection spectrum of 2D SENSI"

plot(chemical_shift,bnmr[i,], type="l", col=color_number,xlim=limx,ylim=limy,main=title, xlab="chemical shift",ylab="')

$\operatorname{grid}()$

points(chemical_shift[peaklist[, 2]], peaklist[, 1], pch=20, col="maroon")

ANS<-readline("

-------Auto peak pick phase-------

OK? type 'yes' or 'no'.

If you want to fix the $x$ or $y$-axis, please type the ' $x$ ' or ' $y$ '

" )

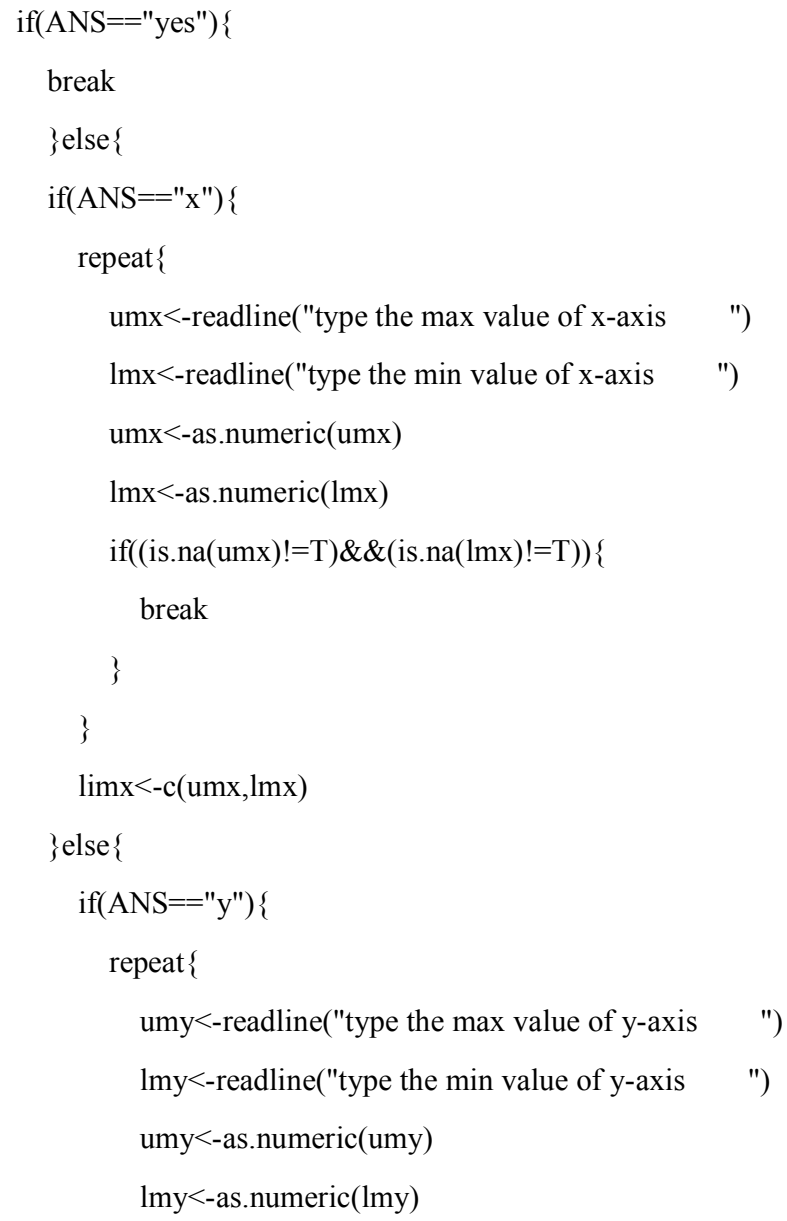




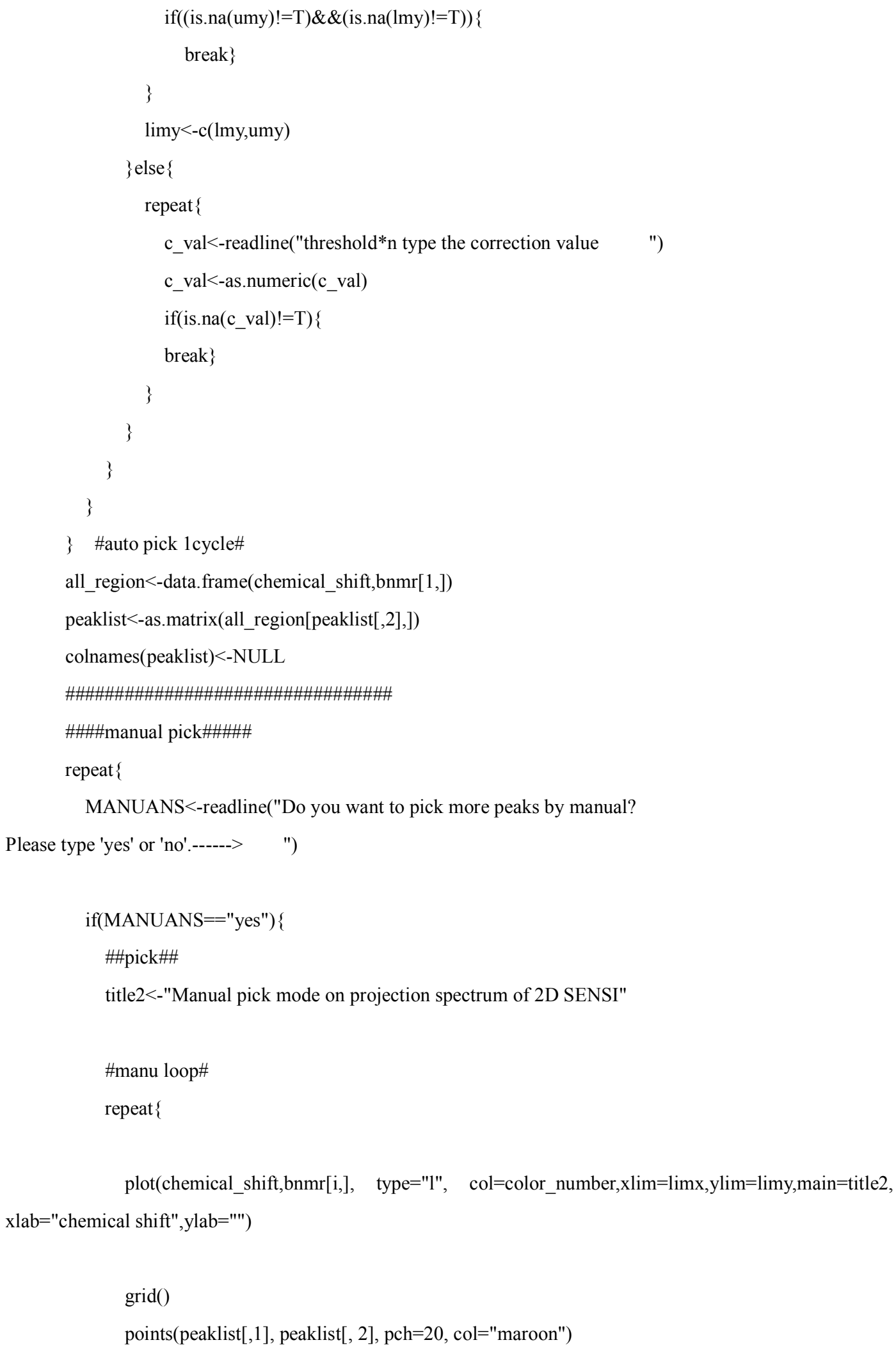




\section{ACTION<-readline("}

please select the next action

' $\mathrm{x}$ '-----> fixing of $\mathrm{x}$-axis

'y'-----> fixing of $x$-axis

'pick'-----> peak pick in this region

'exit'-----> end of manual pick mode

")

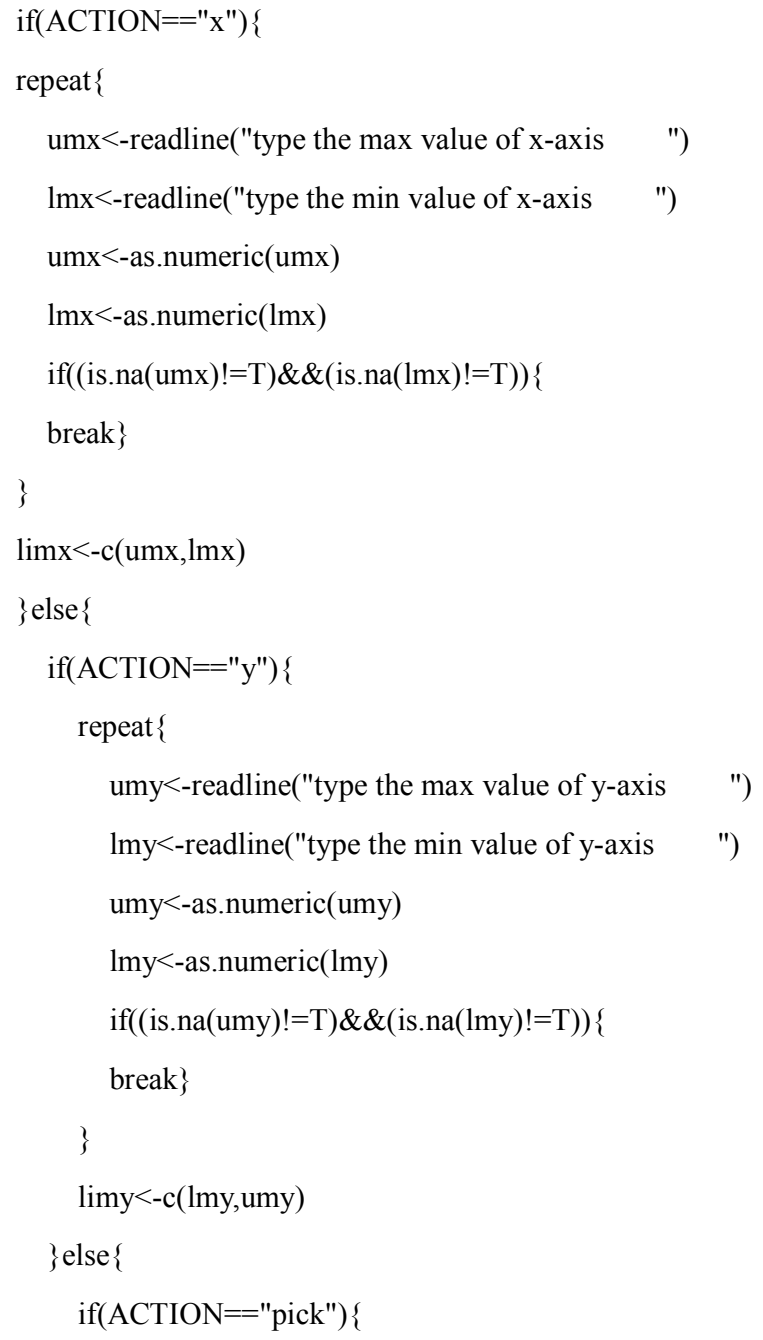




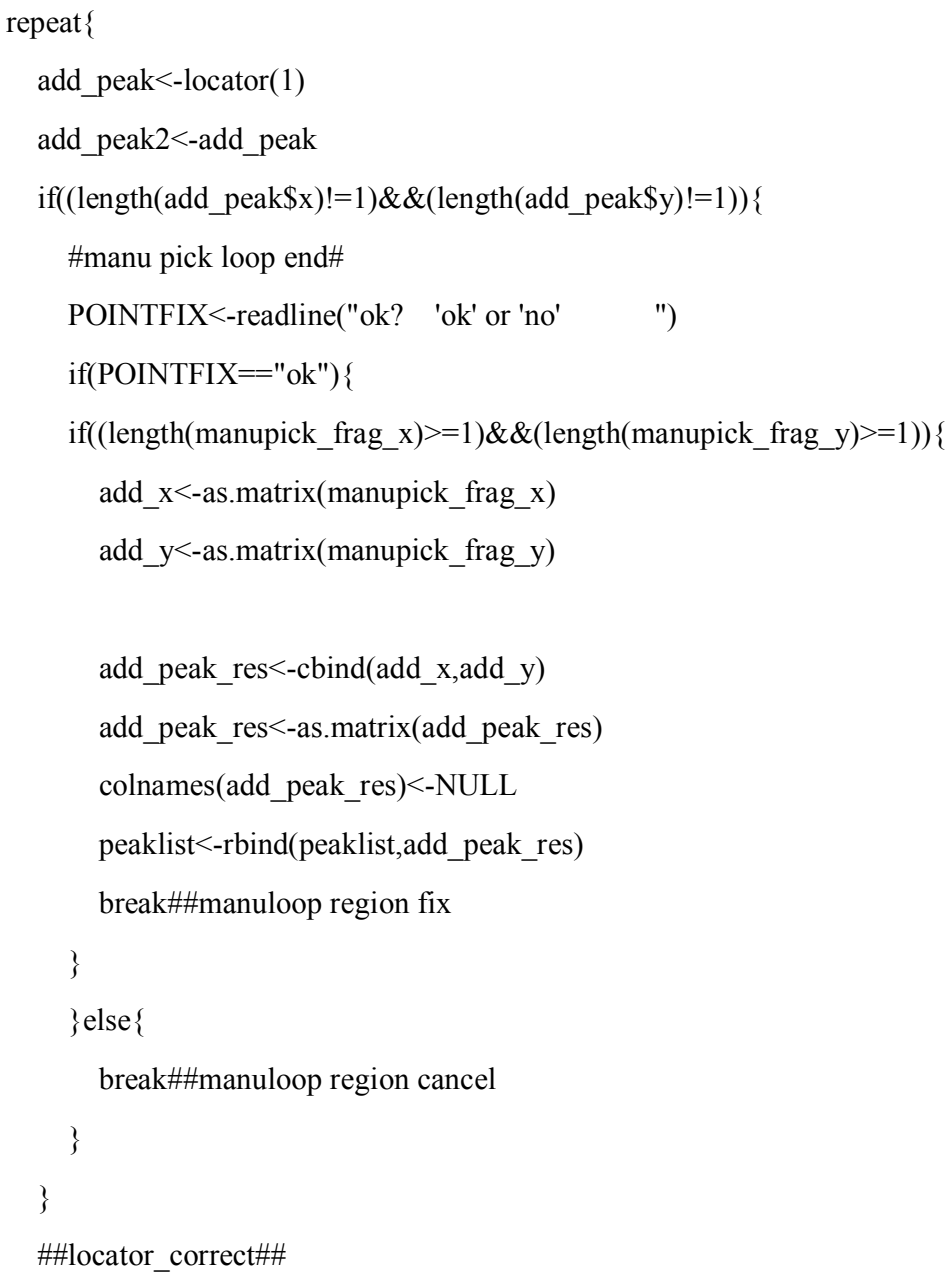

target_region<-all_region[all_region\$chemical_shift $>=($ add_peak\$x-abs(chemical_shift[1]-chemical_shift[6] $))$, ]\#\#minus_correct_value

target_region<-target_region[target_region $\$$ chemical_shift $<=\left(\operatorname{add} \_p e a k \$ x+a b s\left(\operatorname{chemical} \_s h i f t[1]-c h e m i c a l \_s h i\right.\right.$ $\mathrm{ft}[6]))$,]\#\#plus_correct_value

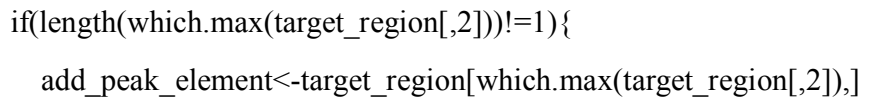




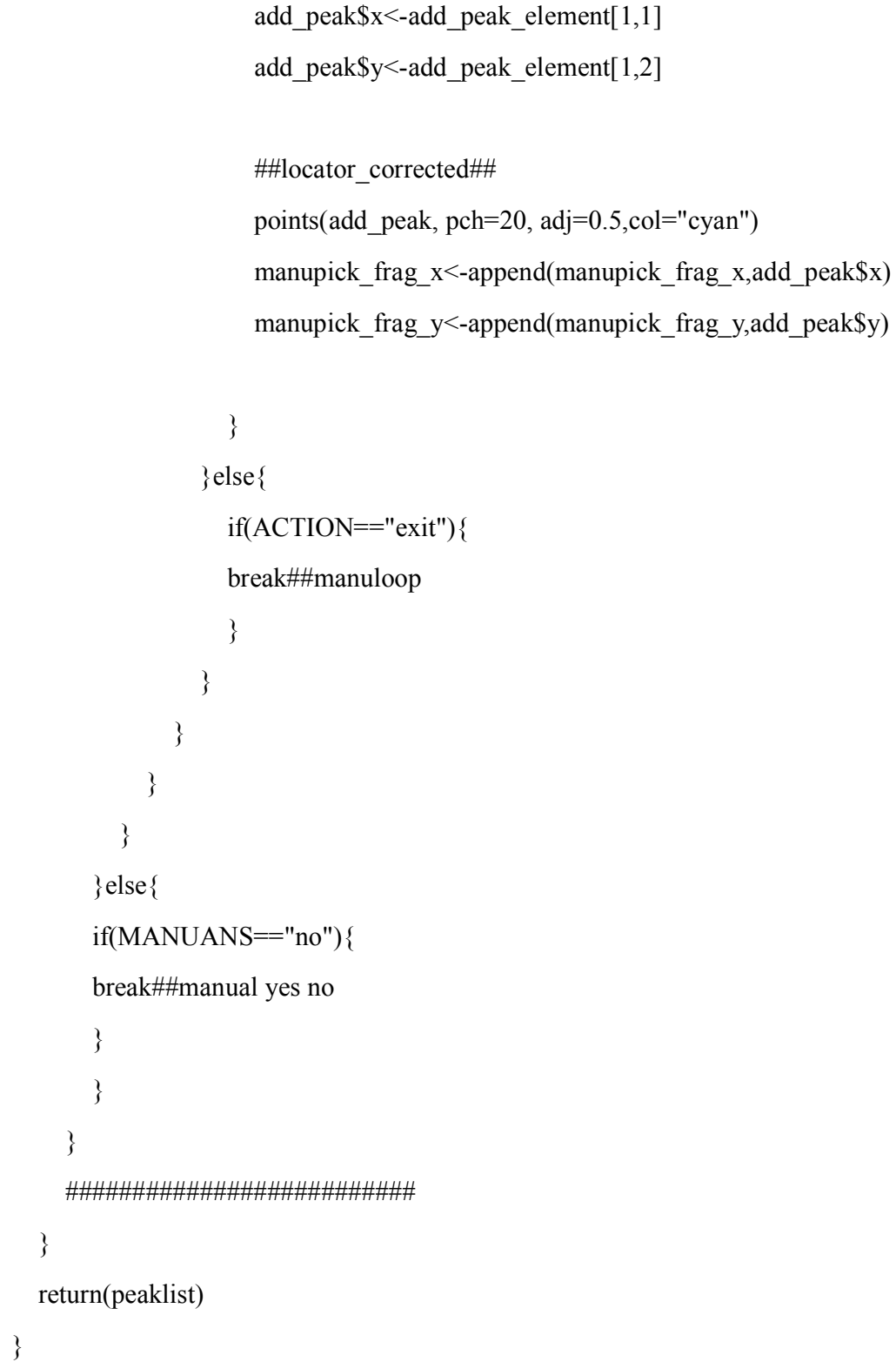




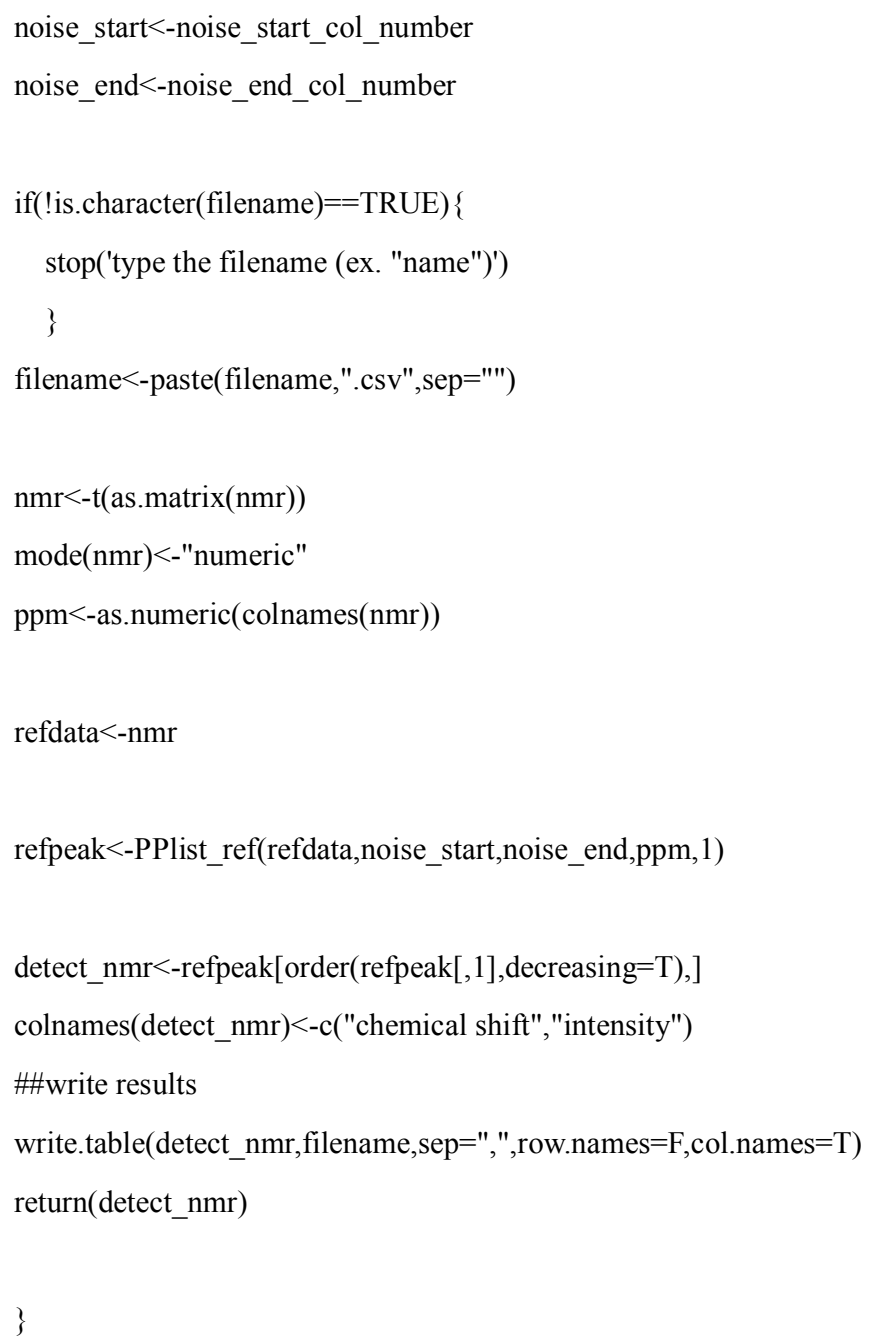




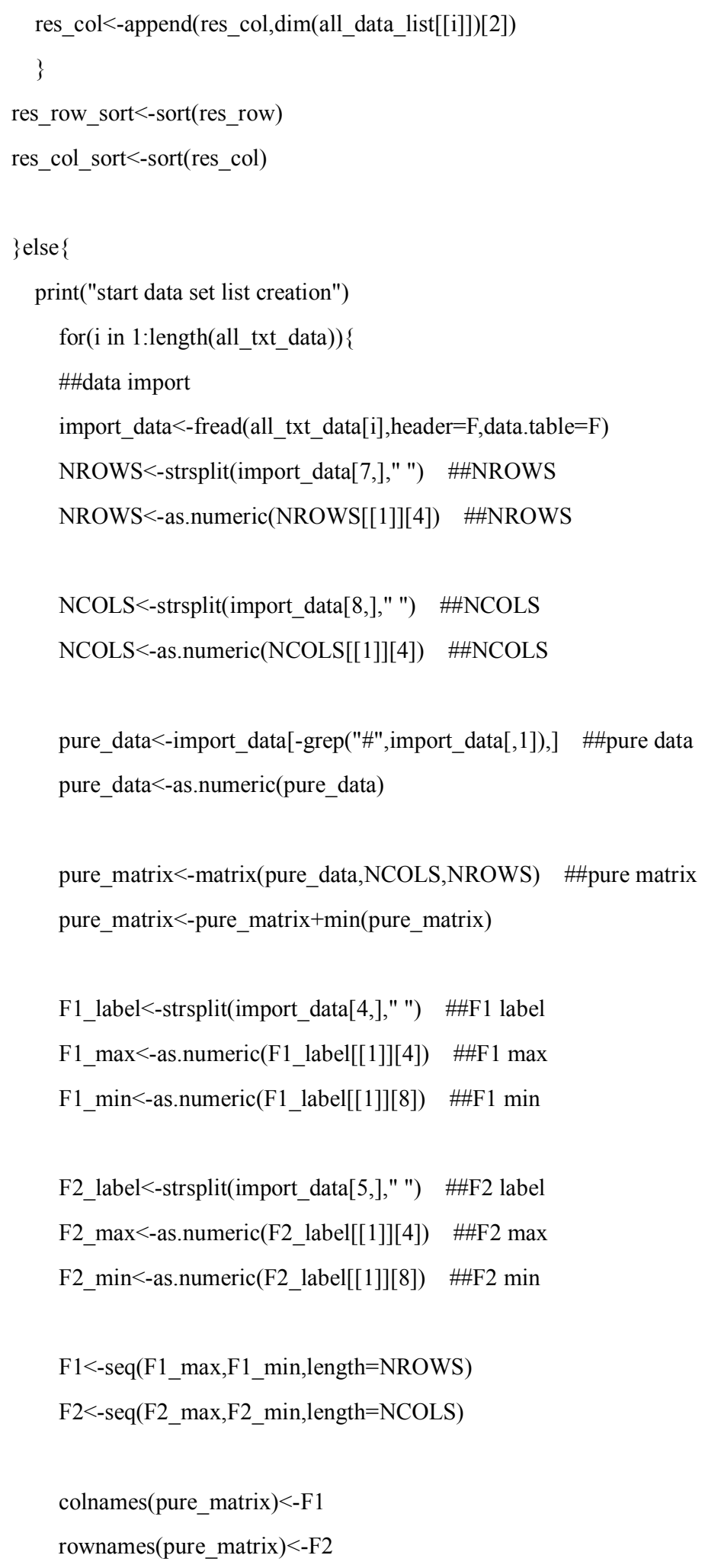




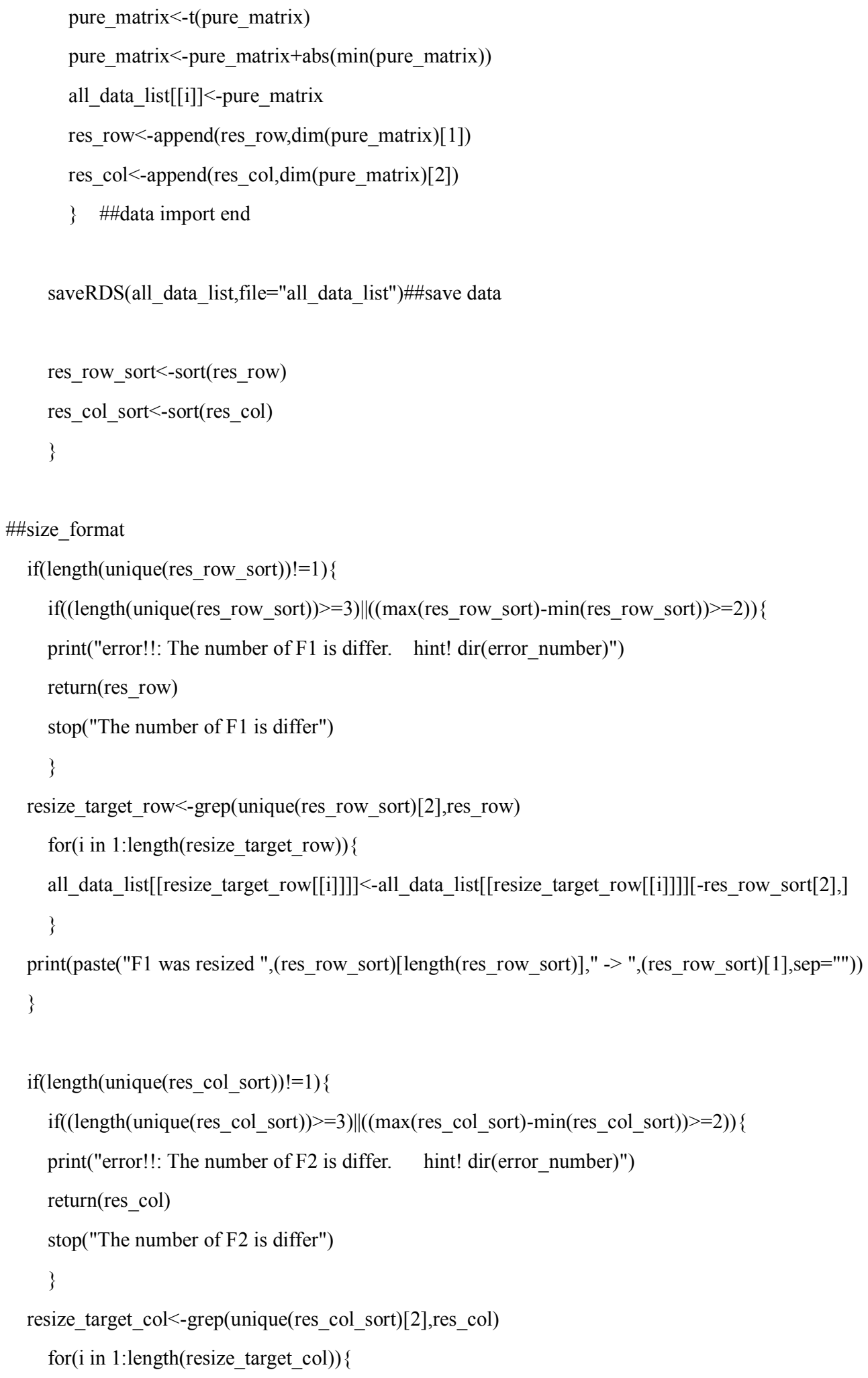




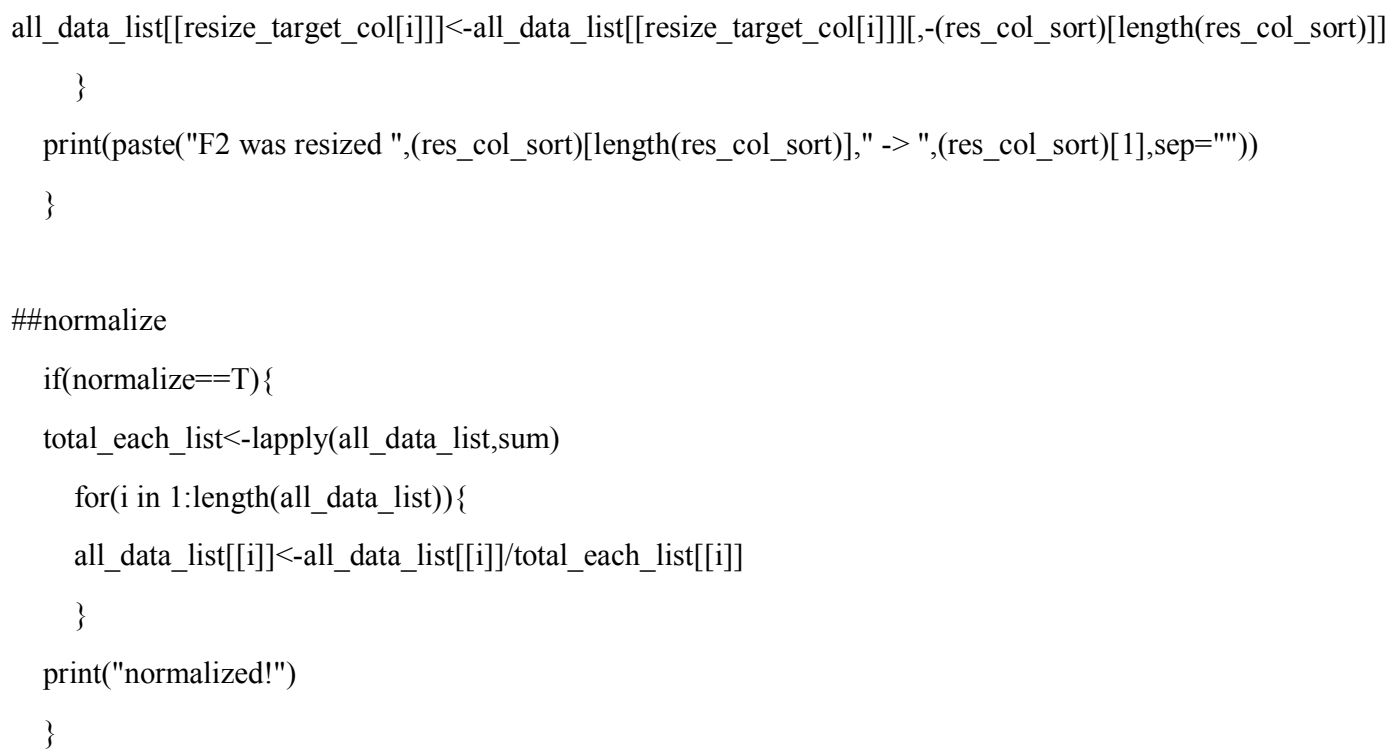




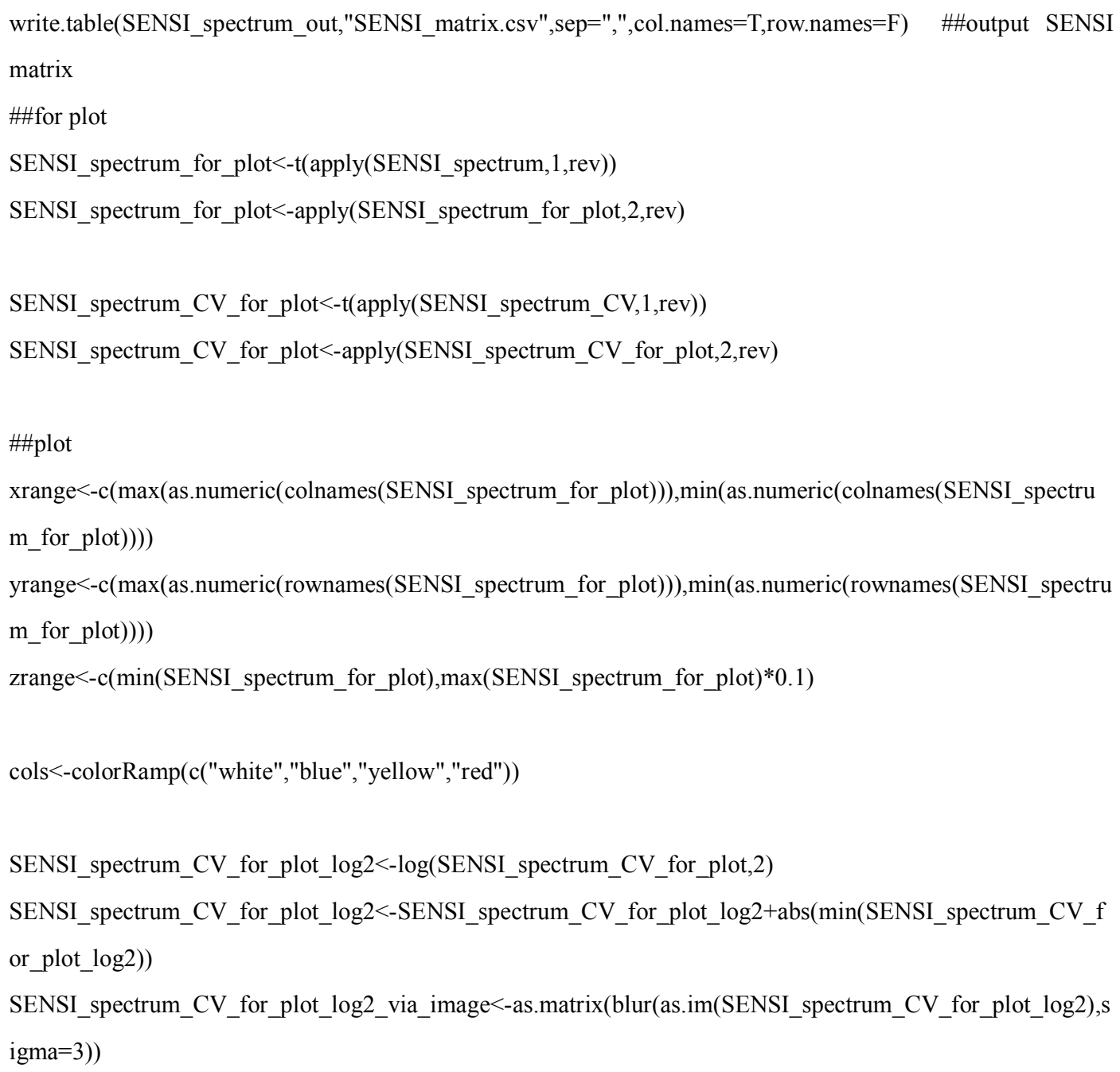




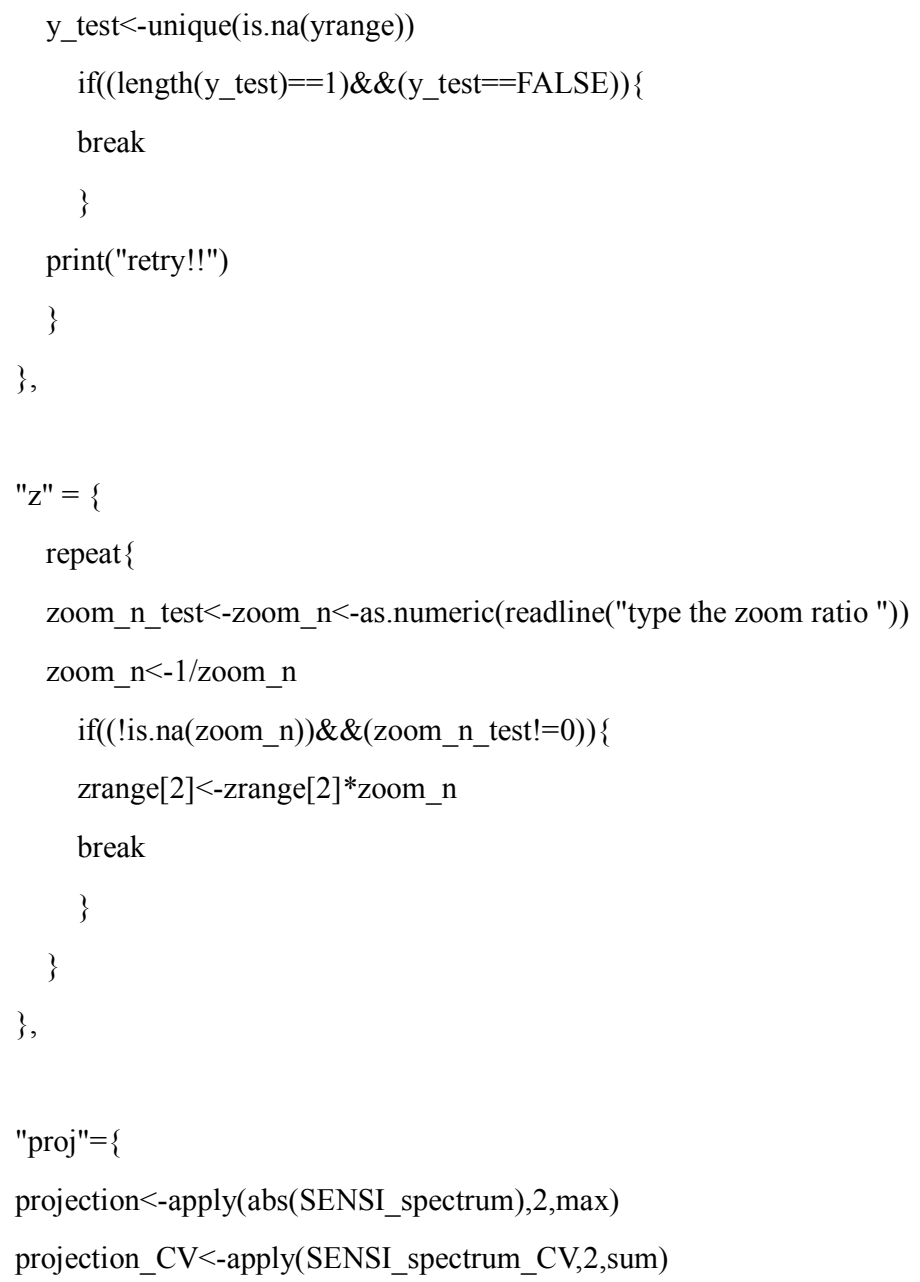

SENSI_proj_DF<-as.data.frame(cbind(as.numeric(colnames(SENSI_spectrum)),projection,projection_CV,log( projection_CV,2)))

names(SENSI_proj_DF)<-c("chemical_shift","intensity","total_of_CV","log2_total_of_CV_of_F1") dev.new()

proj_x<-c(max(as.numeric(colnames(SENSI_spectrum) $))$,min(as.numeric(colnames(SENSI_spectrum))) proj_y<-c(min(SENSI_spectrum),max(SENSI_spectrum))

plot_mid<-median(SENSI_proj_DF\$log2_total_of_CV_of_F1)

proj_plot<-ggplot(SENSI_proj_DF,aes(x=chemical_shift,y=intensity))

proj_plot2<-proj_plot+geom_line(aes(color=log2_total_of_CV_of_F1),size $=0.5)+$ ggtitle("Integrated projection spectrum") 
proj_plot3<-proj_plot2+scale_colour_gradient2(low="blue",mid="olivedrab3",high="red",midpoint=plot_mid)

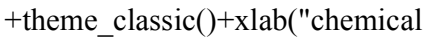
shift")+ylab("

")+theme(axis.title. $x=$ element_text(size $=15)$,axis.title.y=element_text(size $=15))+$ theme $($ axis.text.x $=$ element_te $\mathrm{xt}(\operatorname{size}=15)$,axis.text.y=element_text $(\operatorname{size}=15))$

repeat \{

proj_plot4<-proj_plot3+xlim(proj_x[1],proj_x[2])+ylim(proj_y[1],proj_y[2])

plot(proj_plot4)

proj_ans<-readline("

adjust axis $\rightarrow>$ ' $x$ ' or 'y'

end of projection mode $\quad->\quad$ 'exit'

")

switch(proj_ans,

$" \mathrm{x} "=\{$

repeat \{

proj_x[1]<-as.numeric(readline(" type the max value of $x$-axis "))

proj_x[2]<-as.numeric(readline(" type the min value of $\mathrm{x}$-axis "))

proj_x_test<-unique(is.na(proj_x))

if $(($ length$($ proj_x_test $)==1) \& \&($ proj_x_test $==$ FALSE $))\{$

break

\}

print("retry!!")

\}

\} ,

"y"=\{

repeat \{

proj_y[2]<-as.numeric(readline(" type the max value of y-axis "))

proj_y[1]<-as.numeric(readline(" type the min value of y-axis "))

proj_y_test<-unique(is.na(proj_y))

if $(($ length$($ proj_y_test $)==1) \& \&($ proj_y_test $==$ FALSE $))\{$

break

\}

print("retry!!")

\}

\},

"exit"=\{

write.table(SENSI_proj_DF,"SENSI_proj.csv",col.names=T,row.names=F,sep=",") 


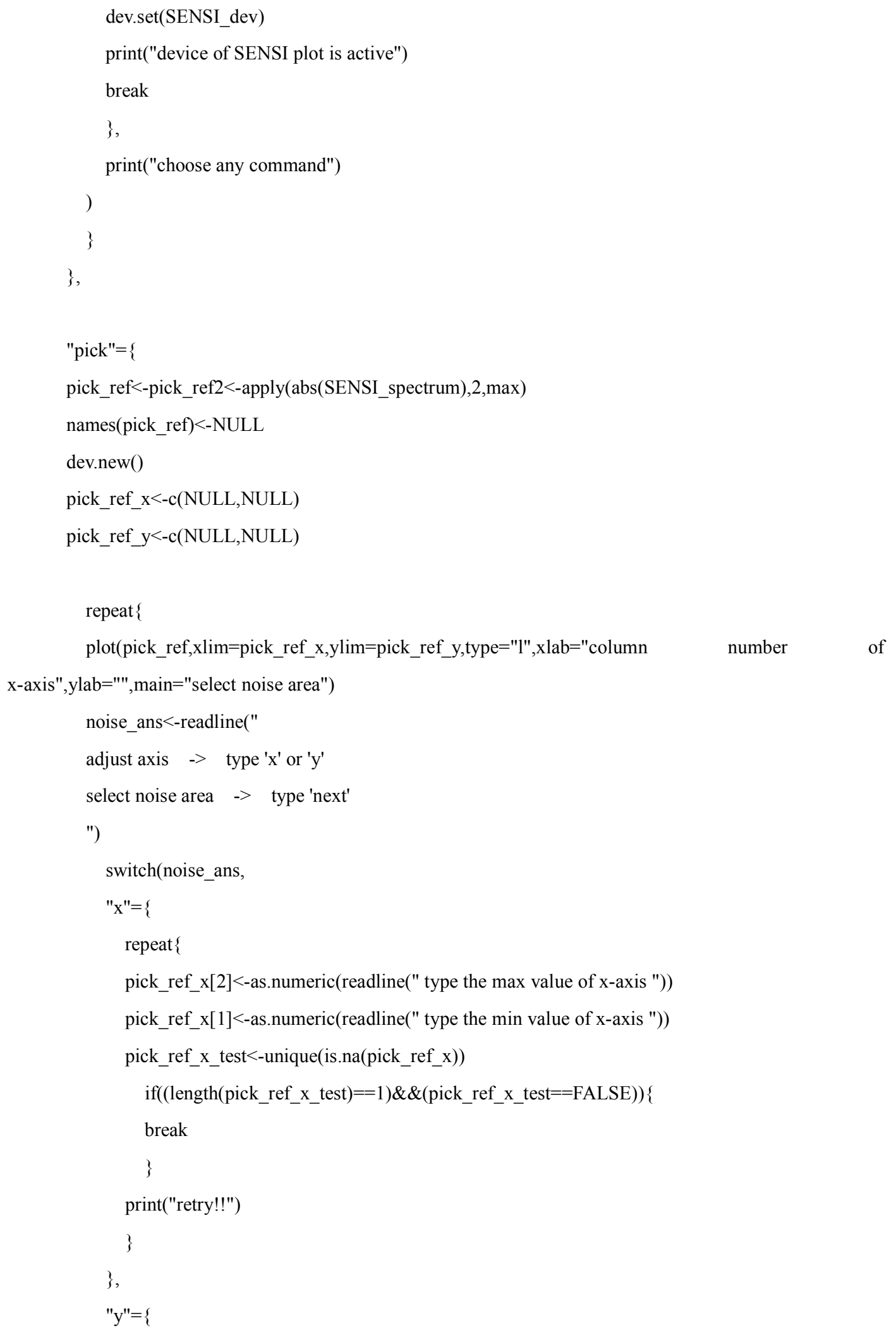




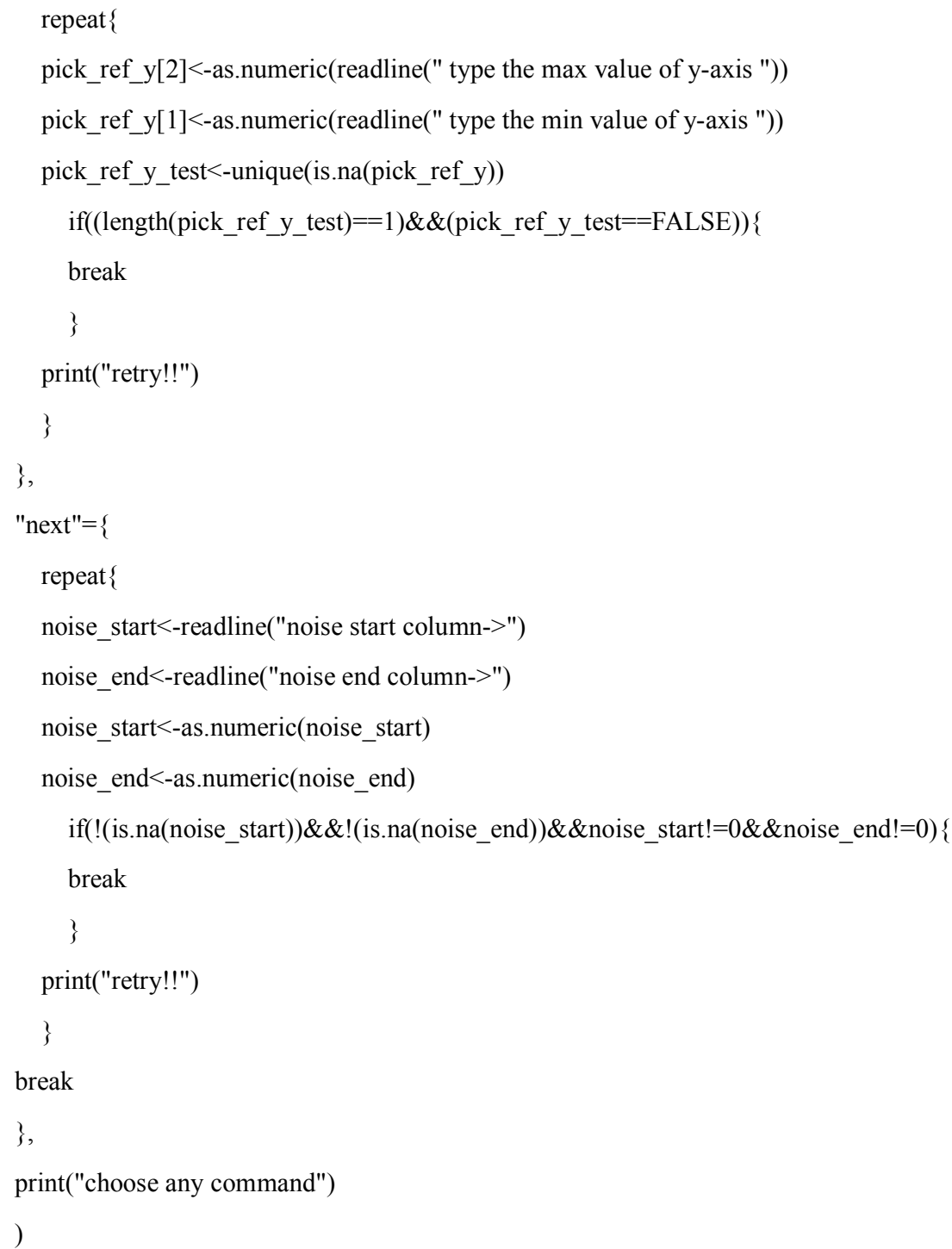




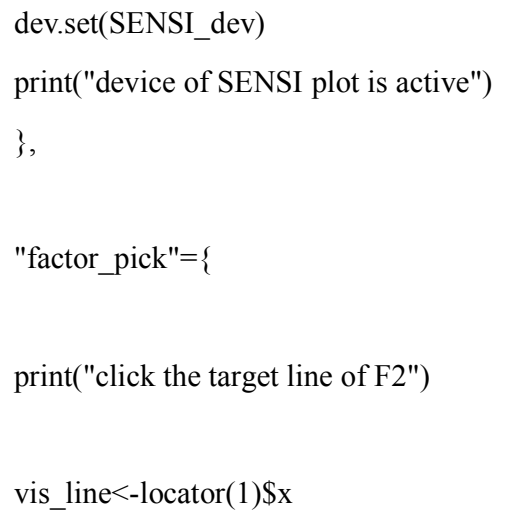

write.table(sep_int_proj,paste("F1_projection_intensity_of_",colnames(SENSI_spectrum)[line_F2],"ppm(F2).c sv",sep="'),row.names=F,col.names=T,sep=",") 
\},

"check"= \{

print("click the target peak")

calc_margin_F1 $<-0.001 \quad$ \#\#enable to adjust calc magin F1 (ppm)

calc_margin_F2 $<-0.005$ \#\#enable to adjust calc magin F2 (ppm)

vis_point $<$-locator $(1)$

polygon(c)(vis_point\$x-calc_margin_F2,vis_point\$x-calc_margin_F2,vis_point $\$ \mathrm{x}+\mathrm{calc} \_$margin_F2,vis_point $\$ \mathrm{x}$ + calc_margin_F2),c(vis_point\$y-calc_margin_F1,vis_point\$y+calc_margin_F1,vis_point\$y+calc_margin_F1,v is_point\$y-calc_margin_F1),col=rgb $(0,0,0$, alpha $=0.5)$, lty $=3$, border $="$ black")

check_F2<-which(abs(as.numeric(colnames(SENSI_spectrum))-vis_point $\$ \mathrm{x})==\min (\operatorname{abs}($ as.numeric(colnames( SENSI_spectrum))-vis_point $\$ \mathrm{x})$ ))

check_F2_low $<-$ which(abs(as.numeric(colnames(SENSI_spectrum))-(vis_point\$x-calc_margin_F2)) $==\min (\mathrm{ab}$ s(as.numeric(colnames(SENSI_spectrum))-(vis_point\$x-calc_margin_F2))))

check_F2_high $<-$ which(abs(as.numeric(colnames(SENSI_spectrum))-(vis_point $\$ \mathrm{x}+$ calc_margin_F2) $==\min (\mathrm{a}$ bs(as.numeric(colnames(SENSI_spectrum))-(vis_point\$x+calc_margin_F2))))

check_F1<-which(abs(as.numeric(rownames(SENSI_spectrum) $)$-vis_point $\$ y)==\min (\operatorname{abs}($ as.numeric $($ rowname s(SENSI_spectrum))-vis_point\$y)))

check_F1_low<-which(abs(as.numeric(rownames(SENSI_spectrum))-(vis_point\$y-calc_margin_F1))==min(a bs(as.numeric(rownames(SENSI_spectrum))-(vis_point\$y-calc_margin_F1))))

check_F1_high<-which(abs(as.numeric(rownames(SENSI_spectrum))-(vis_point\$y+calc_margin_F1))==min( abs(as.numeric(rownames(SENSI_spectrum))-(vis_point\$y+calc_margin_F1))))

check_intensity<-SENSI_spectrum[check_F1,check_F2]

check_CV<-mean(SENSI_spectrum_CV[check_F1_low:check_F1_high,check_F2_low:check_F2_high]) 


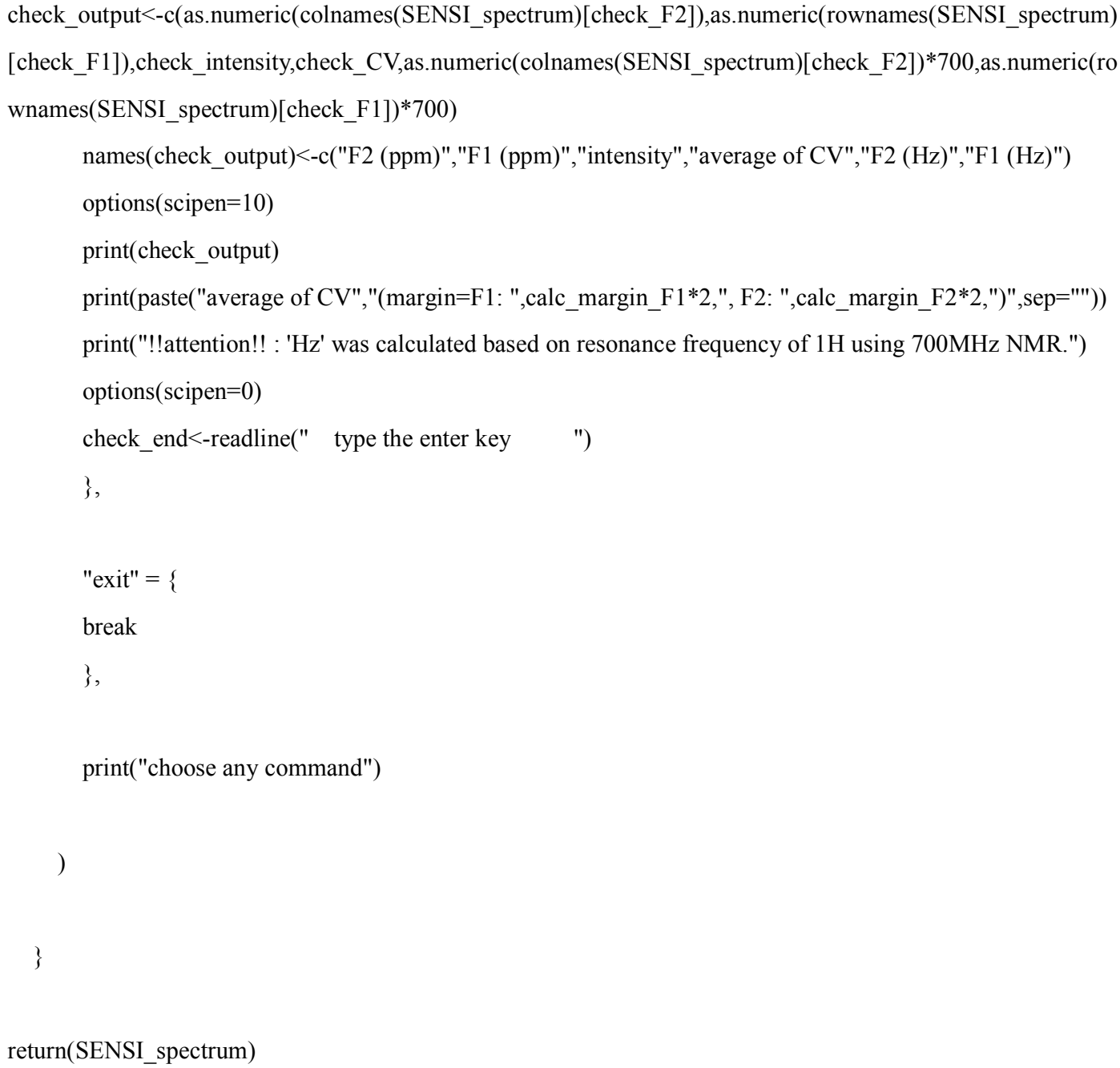

